\title{
Surfactants: materials for interface design and spontaneous formation of structures
}

\author{
C MANOHAR \\ Chemistry Division, Bhabha Atomic Research Centre, Bombay 400085 , India
}

\begin{abstract}
This paper mustly describes the research on surfactant solutions performed at this centre in the past few years. A correlation between microemulsion structure and precipitation reaction of $\mathrm{AgCl}$ is described. Experimental and theoretical research culminating in the development of a theme for analysing the structure of colloidal systems is described. Recent results on the discovery of new viscoelastic surfactant systems are presented.
\end{abstract}

Keywords. Surfactants; cloud point; mixed micelles; viscoelasticity; microemulsions.

\section{Introduction}

Surfactants are molecules with one end hydrophobic and the other hydrophilic (Shah 1977). Because of this they accumulate at interfaces reducing the interfacial tension and also forming various spontaneous structures like monolayers, micelles and microemulsions in water and/or hydrocarbon media (Corti and Degiorgio 1980). These systems resemble structures like the cell membranes of biological systems and hence are of great interest. This article is restricted to three aspects to which we have made contributions, namely microemulsions, clouding phenomenon, and viscoelastic surfactants.

\section{Microemulsions}

Microemulsions are thermodynamically stable, isotropic, transparent mixtures of water, oil and suitably chosen surfactants. Therefore the microemulsion is neither an aqueous nor a nonaqueous liquid which has microstructures on the scale of $\sim 100 \AA$. This system has emerged as a curiosity for physics of novel structures and for chemistry in confined geometries. They have wide-ranging and novel applications which range from self-extinguishable fuel for defence applications to pesticide concentrate formulation in agriculture. They are ideal systems for studying the relation between structure and chemistry.

Figure 1 shows data from some of the studies carried out on the microemulsion of benzene/water/SDS/isopropanol (Leung et al 1985). Two aspects, one structural and the other chemical reactions, were investigated primarily with the idea of understanding the interrelation between the structure and reaction rates. The reaction studied was the precipitation of $\mathrm{AgCl}$ in microemulsion. Two identical microemulsions were prepared, one with $\mathrm{AgNO}_{3}$ and the other with $\mathrm{NaCl}$. These two were mixed in a stop-flow apparatus and the development of turbidity due to precipitation was monitored with time. It was interesting to see that the rate at which the transmittance decreased due to precipitation was dependent on the structure of the medium. The changes in the structure were also reflected in studies of conductivity, ultrasonic 

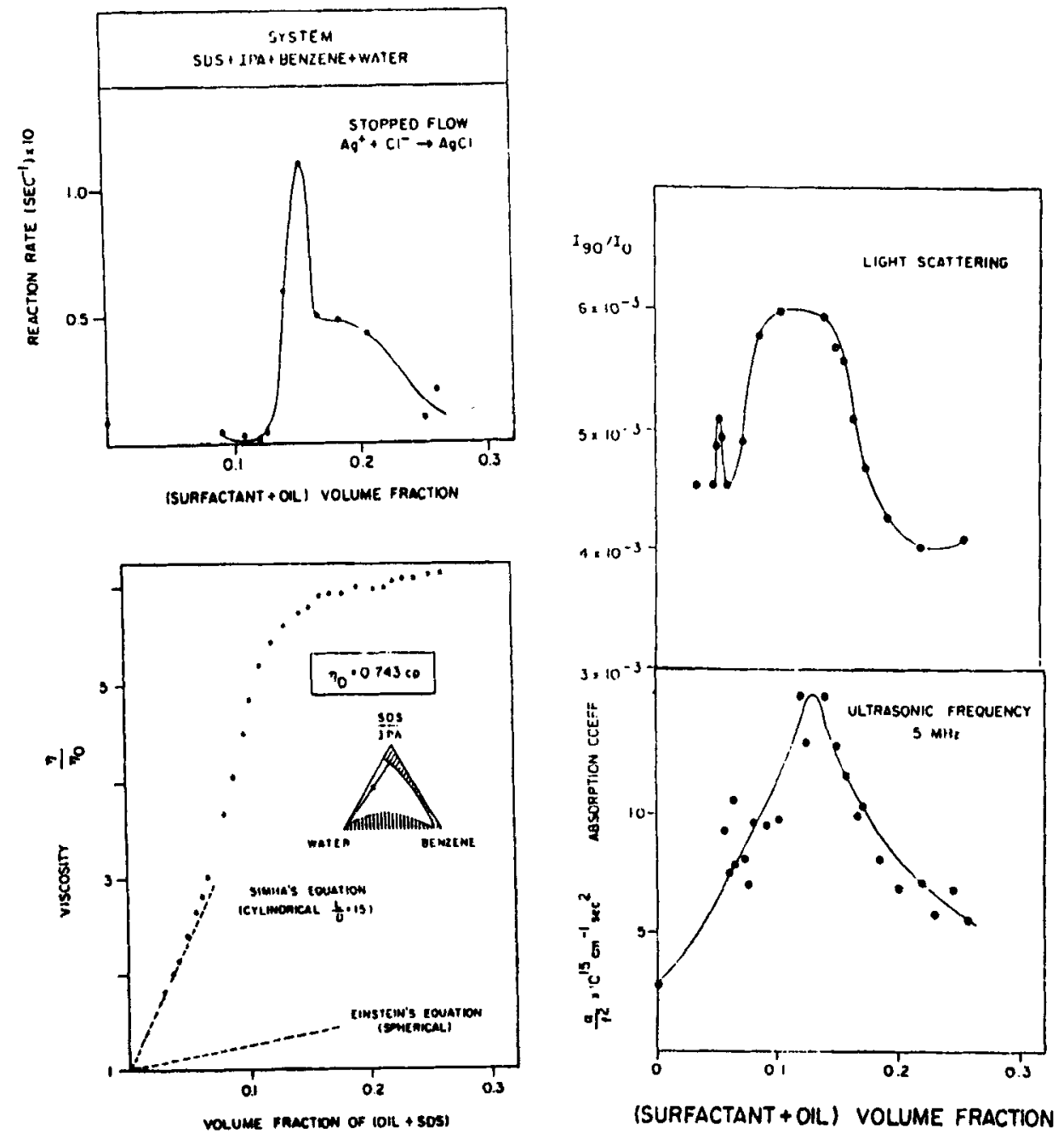

Figure 1. Data for a microemulsion wih the components shown and with weight ratio of SDS/IPA $=0.5$ investigated with several techniques show that around volume fraction of $O I L+S D S=0 \cdot 15$ some structural change occurs which seems to affect the precipitation rates of $\mathrm{AgCl}$.

absorption, etc (figure 1). These studies conclusively showed the relation between chemical reactions and structure in these systems. In recent years these studies have emerged as a way of preparing nanophase materials of a variety of compounds.

\section{Clouding phenomenon}

Nonionic surfactant solutions in water at a level of about $1 \%$ by weight undergo a phase separation into a surfactant-rich phase and a water-rich phase on heating. Associated with this transition is an intense scattering of light as one approaches the phase-separation temperature, the cloud point. This quantity plays an important role in detergency and the mechanism poses an interesting challenge to physicists and 


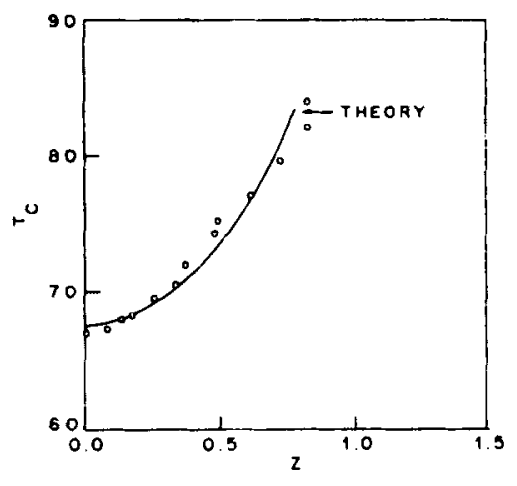

Figure 2. Cloud point measurements of Triton X-100+ SDS shown as the charge on the micelle is varied. The quadratic fit gives the micellar diameter (Manohar and Kelkar 1990) and confirms that the clouding phenomenon is due to interaction between micelles.

chemists. There have been two points of view to understand this phenomenon. One suggests that the surfactant which is water-soluble at room temperature becomes insoluble at higher temperature due to the breaking of hydrogen bonds between the surfactant and water (Mukherjee 1972). The other school suggests that there is an enhanced attraction between the micelles at higher temperatures resulting in a phase separation between micellar phase and water (Corti and Degiorgio 1980). We argued that if the latter is true then any change in the nature of interaction between the micelles should be reflected in a change of the cloud point. We increased the repulsion between micelles of the uncharged surfactant Triton X-100 by incorporating a charged surfactant sodium dodecyl sulphate (SDS) in a controlled way. The cloud point increased, indicating that the interaction between the micelies was important in clouding (Valaulikar and Manohar 1985). Several groups have followed this 'trick' and have come to the same conclusion (Marszall 1988; Gu et al 1989; Huang and $\mathrm{Gu}$ 1990). We developed a semiquantitative model to interpret the rise in cloud point and to obtain micellar diameter from these measurements (Manohar and Kelkar 1990). These results are shown in figure 2. Meanwhile our colleagues at BARC had developed the small angle neutron scattering (SANS) technique and this turned out to be the most suitable for investigating the structure of micellar systems (Desa et al 1985; Goyal et al 1988, 1989). The results obtained for the structure of these systems required not just the semiquantitative models but a more rigorous treatment of the interactions between the micelles. The literature showed that there did not exist suitable theories which take into account the attractive interactions accurately. The most popular model was that by Hayter and Penfold (1981) and this was more suitable for systems where Coulomb interactions dominated. We thought that the more appropriate liquid-state theory for attractive interactions was that of Baxter and adopted it for micellar systems with quantitative description of the SANS data in the literature (Menon et al 1991a). The model was put on a more rigorous footing by realizing that the Baxter model can be looked upon as a perturbation expansion in powers of $\Delta / \sigma$, where $\Delta$ is the range of attractive interactions and $\sigma$ the diameter of the micelle (Menon et al 1991b). Since for colloidal systems this ratio was smaller than unity the Baxter model appeared to be the most natural one to use for colloidal systems. The structure factors calculated using our approach and comparison with 


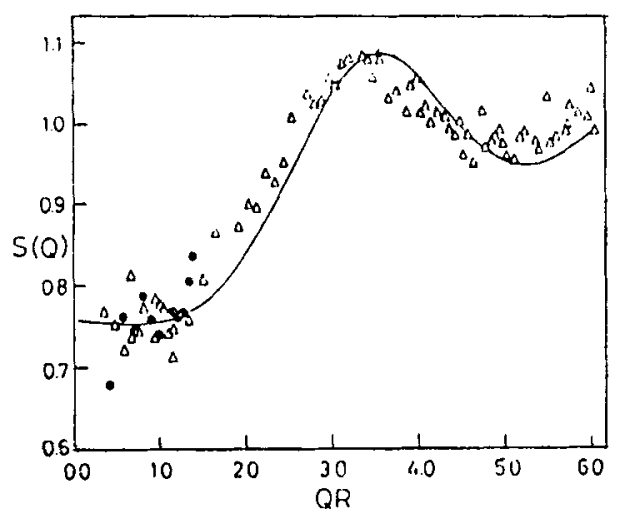

Figure 3. The structure factor calculated (line) by Baxter model without any adjustable parameters (Menon et al 1991) compared with Monte Carlo calculations (circles and triangles) of Huang et al (1984). This case is appropriate for a micellar system.

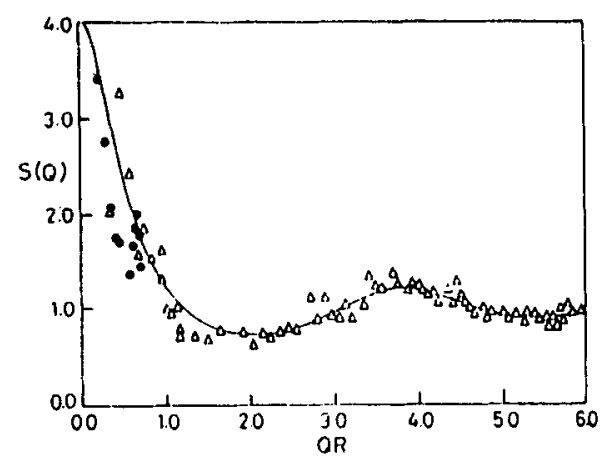

Figure 4. Structure factor similar to figure 3 but this case is more appropriate to a microemulsion.

the Monte Carlo simulations are shown in figures 3 and 4. Once this was realized the need to extend the Baxter model to include the Coulomb interactions became necessary because the majority of the systems in nature are charged systems. We used the Baxter model as the zeroth-order solution to the Ornstein-Zernicke equations and treated the Coulomb interaction in the random phase approximation (Manohar and Kelkar 1992; Kelkar et al 1992). Fortunately, just at that time some French scientists had discovered an ionic surfactant which showed clouding and they had failed to describe their SANS experiments by the existing theories of Hayter and Penfold (Warr et al 1990). It turned out that our Baxter-RPA model was able to fit these data quantitatively with ease (Manohar and Kelkar 1992). Meanwhile experiments on SANS and cloud point measurements had been initiated on two systems, namely a mixture of sodium salicylate (SS) + Triton X-100 and SDS + Triton $\mathrm{X}-100$. The former system, in particular, was extremely neat because one could combine the chemistry and physics beautifully to tune the intermicellar interactions just by tuning the $\mathrm{pH}$ of the system (Manohar et al 1990) and the system was amenable for quantitative interpretation with minimum parameters. The cloud point 
measurements and the fits to results of calculations are shown in figure 5 . The model turned out to be good for this situation. The second system of SDS + Triton X-100 too was found to be quantitatively describable by the Baxter-RPA model we had put forward (Kelkar et al 1991). In this system we also observed the phenomenon of

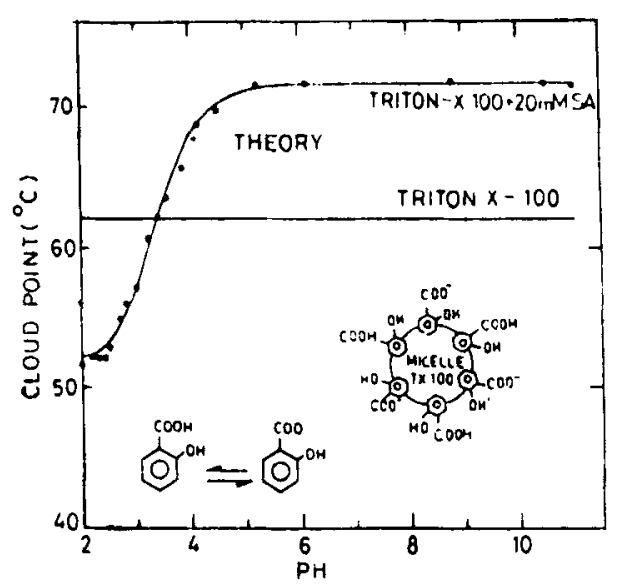

Figure 5. Cloud point altered by tuning the relative strengths of van der Waals and Coulomb interadtions by changing the $\mathrm{pH}$ of the system, and the equations calculated using the interaction between the micelles (Manohar et al 1990): a beautiful combination of chemistry and physics.

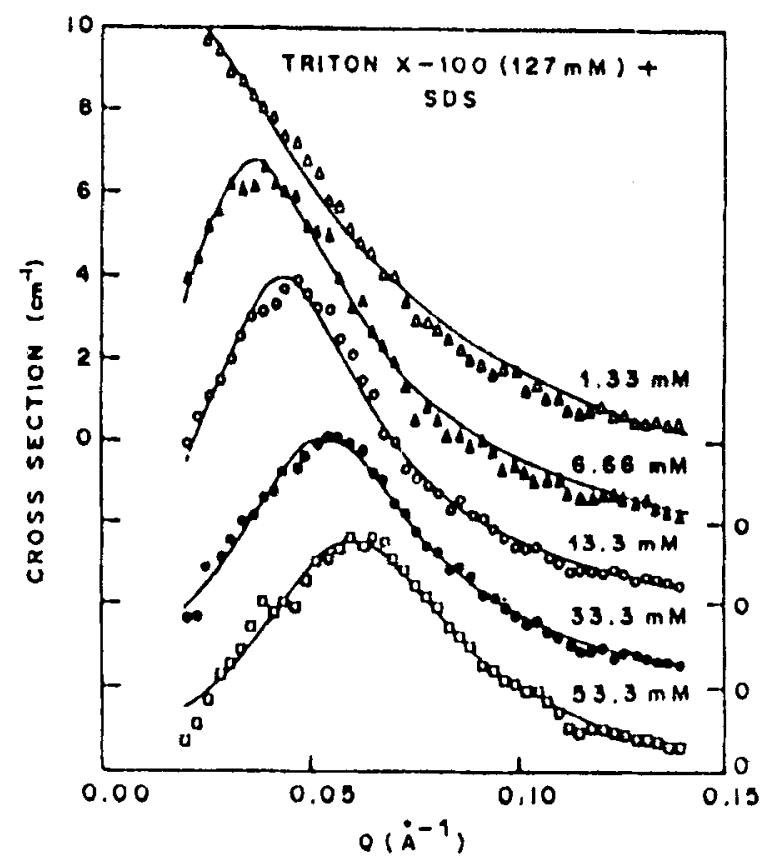

Figure 6. SANS results on Triton $X-100(127 \mathrm{mM})+\mathrm{SDS}$, concentrations shown, and Baxter-RPA model (Manohar and Kelkar 1992) calculations denoteá by lines (Kelkar et al 1991). 
charge condensation on micellar surface. The experimental results of SANS and the result of calculations of RPA-Baxter model are shown in figure 6. On the whole the investigations on the clouding phenomenon turned out to be exciting with the emergence of a theme for description of colloidal systems.

\section{Viscoelasticity}

Cationic surfactants like cetyltrimethylammonium bromide (CTAB) show the interesting phenomenon of drastic increase in viscosity and even elasticity on addition of additives like SS. Such systems have been of great interest and have been investigated in the literature. These are called by various names, e.g. worm-like micelles, thread-like micelles, living polymers. Some aspects of these systems have not been understood, namely the following:

(i) The rise in viscosity shows specificity. If one shifts the OH group in SS to meta or para position the viscoelastic effects decrease drastically (Manohar et al 1986).

(ii) When one adds additives like SS to CTAB the viscosity shows two peaks as the

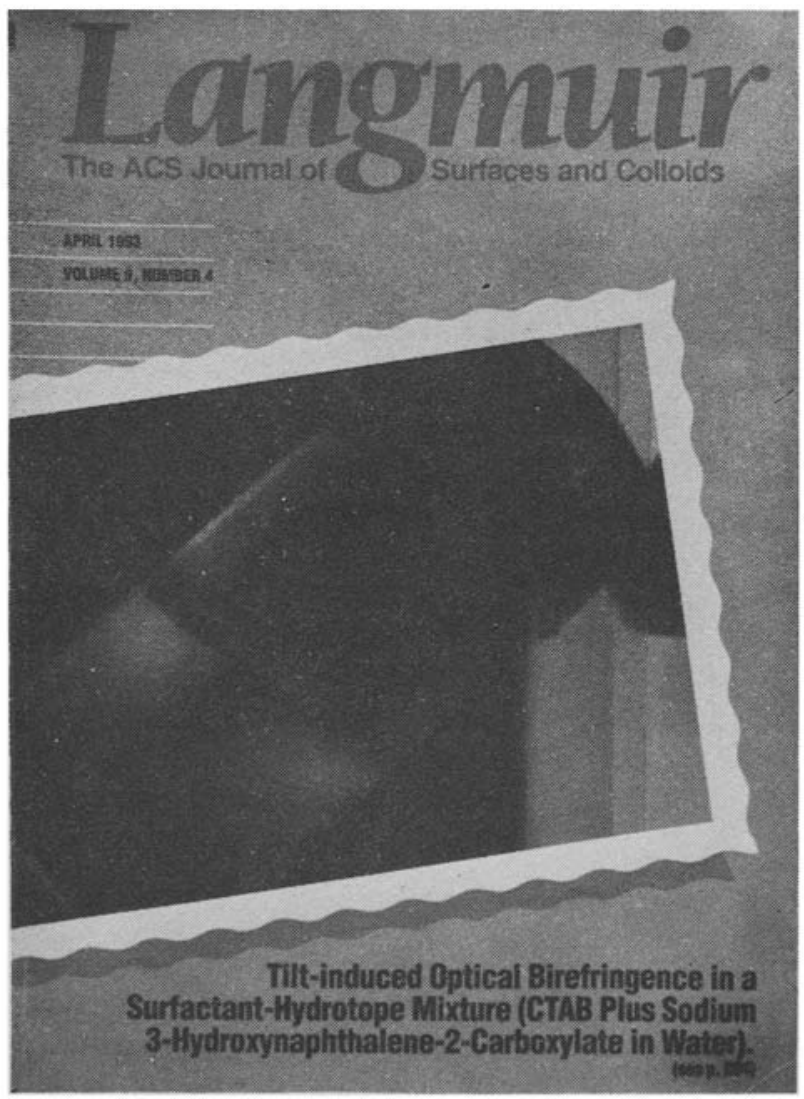

Figure 7. A strongly viscoelastic solution of CTAB and SHNC is observed to show tiltinduced birefringence (Mishra et al 1993). 
concentration of SS is increased keeping the amount of CTAB constant (Rehage and Hoffmann 1991).

(iii) In between the two peaks in the viscosity one observes in some systems phase separations and liquid crystalline phases (Rehage and Hoffmann 1991; Mishra et al 1993).

With a view to understanding the specificity effects we performed NMR experiments on CTAB-SS systems and observed an interesting aspect in the SS part of the NMR spectrum. The SS molecules were adsorbed on micellar surface with their carboxylic groups sticking out in water (Rao et al 1981; Manohar et al 1986). This implied that the SS molecule was surface active! In the literature it had not been recognized that molecules like SS could be surface active. This was further confirmed by other workers in India (Balasubramanian et al 1989). Once this feature was realized we were able to design new systems which were viscoelastic and one with sodium hydroxynaphthalene carboxylate (SHNC) proved to be most exciting (Sushama Mishra et al 1993; Manohar and Janaky Narayanan 1994). This was strongly flow birefringent and just a small tilt in the sample induced the birefringence (figure 7). In this system we observed the nematic phase, which provided the first link between nematics and viscoelastic systems. This aspect is most interesting because nematic liquid-crystalline polymers have been known and it is most likely that the ideas from this field become translatable to surfactant solutions. We are investigating these systems and expecting to derive a lot of excitement in the coming years.

In summary, the developments mentioned above have led not only to just a set of disjointed papers but what has emerged is a theme and, more importantly, a group of scientists interested in surfactants and recognized in international circles.

\section{Acknowledgements}

The above work was collaborative and I would like to thank my friends Prof. D O Shah, University of Florida, for introducing me to this exciting field and encouraging me, and Drs B A Dasannacharya, Janaky Narayanan, V K Kelkar, P S Goyal, S V G Menon, B K Mishra, K S Rao, S D Samant, Sushama Mishra and B S Valaulikar for participation in this excitement.

\section{References}

Balasubramanian D, Srinivas V, Gaikar V G and Sharma M M 1989 J. Phys. Chem. 923865

Corti M and Degiorgio V 1980 Phys. Rev. Lett. 451045

Desa J A E, Majumder S, Sequeira A S and Dasannacharya B A 1985 Solid State Phys. (India) C28 318

Goyal P S, Desa J A E, Narasimhan S L, Dasannacharya B A, Valaulikar B S, Kelkar V K and Manohar C 1988 Mater. Sci. Forum 27163

Goyal P S, Chakravarthy R, Dasannacharya B A., Desa J A E, Kelkar V K, Manohar C, Narasimhan S L, Rao K R and Valaulikat B S 1989 Physica B156 \& 157471

Gu T, Qin S and Ma C 1989 J. Colloid. Int. Sci. 127586

Hayter J B and Penfold J 1981 Mol. Phys. 42109

Huang J S, Saffran S A, Kim M W, Grest G S, Kotlarchyk M and Quirke M 1984 Phys. Rev. Lett. 53592

Huang Z and Gu T 1990 J. Colloid Int. Sci. 138582

Kelkar V K, Mishra B K, Rao K S, Goyal P S and Manohar C 1991 Phys. Rev. A44 8421

Kelkar V K, Janaky Narayanan and Manohar C 1992 Langmuir 82210

Leung R, Hou M J, Manohar C, Shah D O and Chun P W 1985 in Macro-microemulsions: Theory and 
applications (ed.) D O Shah (Gainesville: American Chemical Society) ACS Symposium Ser. 272 p. 235

Manohar C. Rao U R K, Valaulikar B S and lyer R M 1986 J. Chem. Soc. Chem. Comm. 379

Manohar C and Kelkar V K 1990 J. Colloid Int. Sci. 131604

Manohar C, Kelkar V K, Mishra B K, Rao K S, Goyal P S and Dasannacharya B A 1990 Chem. Phys. Lett. 171451

Manohar C and Kelkar V K 1992 Langmuir 818

Manohar C and Janaky Narayanan 1994 Phase Transitions 50125

Marszall L 1988 Langmuir 490

Menon S V G, Kelkar V K and Manohar C 1991a Phys. Rev. A43 1130

Menon S V G, Rao K S and Manohar C 1991b J. Chem. Phys. 959186

Mishra B K, Samant S D, Pradhan P, Sushama B Mishra and Manohar C 1993 Langmuir 9894

Mukherjee P 1972 J. Phys. Chem. 76565

Rao U R K, Manohar C, Valaulikar B S and Iyer R M 1980 J. Phys. Chem. 913286

Rehage H and Hoffmann H 1991 Mol. Phys. 74933

Shah D O 1977 Chem. Engg. Education 1114

Sushama B Mishra, Mishra B K, Samant S D, Janaky Narayanan and Manohar C 1993 Langmuir 92804

Valaulikar B S and Manohar C 1985 J. Colloid Int. Sci. 108403

Warr G G, Zemb T N and Drifford M 1990 J. Phys, Chem. 943086 